\title{
Verhaltenstherapie
}

\section{Band 13, Heft 4, Dezember 2003}

\section{Editorial}

233 Auf ein Wort: Zur kommunikativen Bedeutung des Wortes «eigentlich» in der Psychotherapie Fichter, M.M. (Prien)

236 Dank an die Gutachter

Originalarbeiten

237 Wirksamkeit und Prozessmerkmale einer psychoedukativen und bewältigungsorientierten Gruppentherapie für schizophren und schizoaffektiv Erkrankte

Fries, A.; Andres, K.; Pfammatter, M.; Brenner, H.D. (Bern)

244 Der Einfluss von psychologischer Psychotherapie auf beängstigenden Schwindel bei Panikstörung mit Agoraphobie

Heinrichs, N.; Hahlweg, K.; Moschner, C.; Wessel, K.; Fiegenbaum, W. (Braunschweig)

254 Das Gruppenprogramm «Depression bewältigen» und seine Varianten - eine aktualisierte Metaanalyse Kühner, C. (Mannheim)

\section{Übersichtsarbeiten}

264 Achtsamkeit («Mindfulness») als Therapieprinzip in Verhaltenstherapie und Verhaltensmedizin Heidenreich, T. (Frankfurt/M.); Michalak, J. (Bochum)

276 Zur Psychotherapie der Aufmerksamkeitsdefizit- I Hyperaktivitätsstörung (ADHS) bei Erwachsenen Heßlinger, B.; Philipsen, A.; Richter, H.; Ebert, D. (Freiburg i.Br.)

Aus der Praxis für die Praxis

284 Zusammenarbeit einer Spezialambulanz mit niedergelassenen Allgemeinmedizinern und Nervenärzten bei der Behandlung von Angsterkrankungen Wittmund, B.; Wilms, H.-U.; Bull, N.; Angermeyer, M.C. (Leipzig)
Vol. 13, Issue 4, December 2003

Editorial

233 Remarks on the Communicative Meaning of the Word 'eigentlich' in Psychotherapy Fichter, M.M. (Prien)

236 Acknowledgement to the Reviewers

Original Articles

237 Outcome and Process Characteristics of a Psychoeducational, Coping Oriented Group Therapy for Schizophrenia and Schizoaffective Patients Fries, A.; Andres, K.; Pfammatter, M.; Brenner, H.D. (Bern)

244 The Impact of Psychological Psychotherapy on Anxiety Provoking Dizziness in Panic Disorder with Agoraphobia Heinrichs, N.; Hahlweg, K.; Moschner, C.; Wessel, K.; Fiegenbaum, W. (Braunschweig)

254 The Coping with Depression Course and Its Variants an Updated Meta-Analysis Kühner, C. (Mannheim)

Review Articles

264 Mindfulness as a Treatment Principle in Behaviour Therapy

Heidenreich, T. (Frankfurt/M.); Michalak, J. (Bochum)

276 Psychotherapy of Attention Deficit Hyperactivity Disorder in Adults

Heßlinger, B.; Philipsen, A.; Richter, H.; Ebert, D. (Freiburg i.Br.)

For the Practitioner

284 Cooperation of a Specialized Outpatient Unit, General Practitioners and Psychiatrists in the Treatment of Anxiety Disorders Wittmund, B.; Wilms, H.-U.; Bull, N.; Angermeyer, M.C. (Leipzig)

\begin{tabular}{ll}
\hline KARGER & @ 2003 S. Karger GmbH, Freiburg \\
Fax +49 761 45207 14 & Artikel (Volltext) und Inhaltsverzeichnisse \\
$\begin{array}{l}\text { E-mail Information@Karger.de } \\
\text { www.karger.com }\end{array}$ & sowie das vorläufige Inhaltsverzeichnis des nächsten Heftes: \\
www.karger.com/ver_bk.htm
\end{tabular}




\section{Verhaltenstherapie}

\section{Band 13, Heft 4, Dezember 2003}

Diskussionsforum

291 Qualitätsmonitoring in der ambulanten Psychotherapie: Modellprojekt der Techniker Krankenkasse Fydrich, T. (Heidelberg); Nagel, A. (Hamburg); Lutz, W. (Bern), Richter, R. (Hamburg)

296 Wissenschaftliche Stellungnahme zu: Qualitätsmonitoring in der ambulanten Psychotherapie: Modellprojekt der Techniker Krankenkasse. Verhaltenstherapie 2003;13:291-295

Schulte, D. (Bochum)

297 Anmerkungen zu: Qualitätsmonitoring in der ambulanten Psychotherapie: Modellprojekt der Techniker Krankenkasse. Verhaltenstherapie 2003;13:291-295

Engelhardt, W. (Überherrn-Bisten)

Interview

299 Maren Langlotz-Weis: «Familienaufstellung in der Verhaltenstherapie - Erweiterung des Repertoires oder Modeerscheinung?»

303 Neuigkeiten vom Buch- und Zeitschriftenmarkt

307 Fort- und Weiterbildung

310 Mitteilungen der Verbände

315 Tagungen und Kongresse

318 Autorenverzeichnis Band 13, 2003

319 Sachwortverzeichnis Band 13, 2003

III Inhalt Band 13, 2003 (nach Seite 322)

298 Impressum

321 Hinweise für Autoren

U3 Erläuterungen zum Titelbild (3. Umschlagseite) Leibl, C.; Gottschalk, A.; Sutner, L. (Prien)
Vol. 13, Issue 4, December 2003

Forum

291 Quality Monitoring in Outpatient Psychotherapy: Pilot Project of the 'Techniker Krankenkasse' Fydrich, T. (Heidelberg); Nagel, A. (Hamburg); Lutz, W. (Bern), Richter, R. (Hamburg)

296 Scientific Remarks on: Quality Monitoring in Outpatient Psychotherapy: Pilot Project of the 'Techniker Krankenkasse'. Verhaltenstherapie 2003; 13:291-295

Schulte, D. (Bochum)

297 Comments on: Quality Monitoring in Outpatient Psychotherapy: Pilot Project of the 'Techniker Krankenkasse'. Verhaltenstherapie 2003;13:291-295 Engelhardt, W. (Überherrn-Bisten)

Interview

299 Maren Langlotz-Weis: 'Family Constellation in Behavior Therapy - Expansion of Methods or Temporary Fashion?'

303 Articles and Books

307 Education

310 Information by Behavior Therapy Associations

315 Meetings and Conferences

318 Author Index Vol. 13, 2003

320 Subject Index Vol. 13, 2003

V Contents Vol. 13, 2003 (following page 322)

298 Imprint

322 Guidelines for Authors

U3 Explanations on the Title (Inside back cover) Leibl, C.; Gottschalk, A.; Sutner, L. (Prien) 